\title{
Semantic information in short-term memory: Effects of presenting recall instructions after the list
}

\author{
N. E. WETHERICK \\ University of Aberdeen, Old Aberdeen, Scotland AB9 $2 U B$
}

\begin{abstract}
Lists of eight words drawn from two or four semantic categories were presented, followed by instructions requiring either free recall, sequential recall (in order of presentation), or semantic recall (in groups of words from the same semantic category). The hypothesis that semantic recall is a retrieval phenomenon while sequential recall depends on attention to sequential characteristics of the lists during input was supported. Presenting recall instructions after the list reduced sequential recall but enhanced semantic recall.
\end{abstract}

Wetherick (1975) presented for immediate recall lists of eight single-syllable words drawn from one, two, four, or eight semantic categories. He found a negative curvilinear relationship between the number of words recalled and the number of semantic categories from which they had to be retrieved, which he interpreted as evidence supporting Craik's "alternative framework" for human memory research (Craik, 1973; Craik \& Lockhart, 1972). He suggested that words may pass directly to corresponding memory traces and that the possibility of retrieval may be affected by preexisting structural relationships between the traces, which may be close (if the words come from one semantic category) or distant (if they come from many). He also suggested that sequential tagging may, in Craik's terms, represent a memory function involving "continued attention to one aspect of the situation" (i.e., ordinal position in the list).

The fact that number of between-category input shifts does not effect recall (as had previously been shown by Sanders \& Schroots, 1968) suggests that the semantic effect may be a retrieval phenomenon. Efficiency of sequential tagging appears, however, to depend on attention to that aspect of the situation during input, since the serial position curve was substantially changed by instructions to recall in order of presentation (early items in the list being recalled better and later items worse).

In Wetherick (1975) each subject worked under one instruction condition which was given in advance. The subject was required to recall words in order of presentation (SR instructions), or in any order (FR instructions), or in any order but with attention drawn to the possibility of semantic relationships between words in the list [SR(C) instructions]. Three separate scores were

This paper is sponsored by Roger L. Dominowski, who takes full editorial responsibility for its contents. obtained: S1 (number of words recalled in order of presentation), $\mathbf{S} 2$ (number of words recalled in any order), and S3 (number of words recalled in groups related in meaning).

S1 scores were calculated by scoring $2,3,4$, or more for every sequence of two, three, four, or more words recalled in order of presentation. S3 scores were calculated by scoring $2,3,4$, or more for every sequence of two, three, four, or more words recalled from the same semantic category. An S3 score could only be obtained on lists presenting either four words from two semantic categories (4-2 lists) or two words from four categories (2-4 lists).

Presenting the recall instructions after the list of words clearly constitutes a test of the hypothesis that semantic recall is a retrieval phenomenon, while sequential recall depends on attention to that aspect of the situation at the input stage. It may be predicted to have no effect on semantic recall but to handicap sequential recall. In the present experiment the hypothesis was submitted to this test.

\section{METHOD}

The same semantic categories were employed as in Wetherick (1975). This time, however, only lists providing S1, S2, and S3 scores were required, so six 4-2 lists were selected consisting of four words from two different categories and six 2-4 lists were selected consisting of two words from four categories. No word was used more than once, and no two successive words in a list came from the same semantic category. The lists were recorded on tape in random order, using a female voice and at a speed of $120 \mathrm{wpm}$.

\section{Instructions}

"This is a test of how well you can remember the words in a list immediately after you have heard the list. You will hear a warning tone like this [tone sounds] and then a list of words followed by a second tone. Some of the words may be linked in meaning. For example, they may be names of flowers or names of places. 
"When you hear the second tone, I shall place a card on the table, here, which says either 'order' or 'meaning' or is blank. If I place a blank card on the table, you may recall the words in any order you like [FR instructions]. If the card says 'order,' you must try to recall the words in the order in which they were presented [SR instructions] . If the card says 'meaning,' you must try to recall the words in groups related in meaning [SR(C) instructions].

"Here is practice list to show you what I mean... Good, here is another practice list... Now we will begin the experiment proper. When you have reported back the words from one list, we will go straight on to the next."

The practice lists consisted of eight unrelated words and were presented with a blank card.

\section{Subjects}

Thirty subjects were employed (18 female, 12 male); all were members of a first-year psychology class. Each subject repeated back 12 lists (six 4-2 and six 2-4 lists; two of each type presented with a blank card, two with an "order" card, and two with a "meaning" card).

\section{RESULTS}

S1, S2, and S3 scores were calculated, and Table 1 shows mean scores for the two types of list and the three types of instruction. The FR and SR instructions had the same meaning as in the earlier study, but were presented after each list, not at the beginning of the experimental session. The $\mathrm{FR}(\mathrm{C})$ instructions were stronger than in the original study, requiring recall in groups of words related in meaning instead of merely suggesting that helpful relationships of this kind might exist. The figures in parentheses in Table 1 are the mean scores obtained in the original study at the same speed. On 4-2 lists (all instructions) and 2-4 lists (FR instructions), the mean S2 score (total number of words recalled) is comparable with that obtained in the original study (using different subjects and lists). On $2-4$ lists (SR and FR(C) instructions), the mean S2 score is lower. For both types of list and all instructions, the mean S1 score (number of words recalled in order) is substantially lower than in the original study, and the mean S3 score (number of words recalled in groups related in meaning) is substantially higher. Presenting the instructions after each list handicaps sequential recall (S1 score), as predicted by the hypothesis, but appears to enhance semantic recall (S3 score).

Table 1

Mean Number of Words Recalled Out of Eight*

\begin{tabular}{lcccccc}
\hline & \multicolumn{2}{c}{ S1 Score } & \multicolumn{2}{c}{ S2 Score } & \multicolumn{2}{c}{ S3 Score } \\
Inst. & \multicolumn{2}{c}{ Type of List } & \multicolumn{2}{c}{ Type of List } & \multicolumn{2}{c}{ Type of List } \\
Cond. & $4-2$ & $2-4$ & $4-2$ & $2-4$ & $4-2$ & $2-4$ \\
\hline \multirow{2}{*}{ SR } & 1.75 & 1.27 & 4.93 & 3.15 & 2.58 & .57 \\
& $(3.32)$ & $(3.60)$ & $(4.93)$ & $(4.76)$ & $(1.64)$ & $(.29)$ \\
FR & 1.43 & 1.53 & 4.63 & 4.57 & 2.63 & 1.15 \\
& $(3.05)$ & $(2.85)$ & $(5.23)$ & $(4.59)$ & $(1.90)$ & $(.58)$ \\
FR(C) & .50 & .37 & 5.53 & 3.48 & 5.32 & 2.02 \\
& $(2.61)$ & $(3.14)$ & $(5.04)$ & $(5.02)$ & $(2.38)$ & $(.52)$ \\
\hline
\end{tabular}

*Figures in parentheses are comparable means from Wetherick (1975).
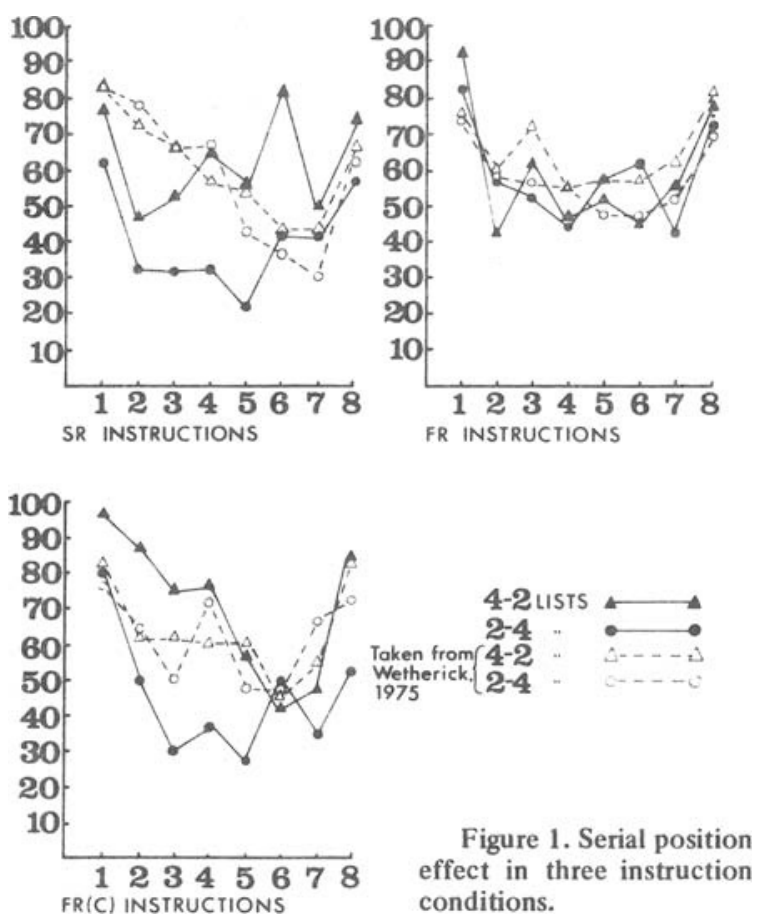

Figure 1 shows serial position curves based on S2 scores under the three instruction conditions. The curves are drawn on a percentage correct basis to allow comparability between this experiment (30 subjects, two lists each) and the equivalent group in the earlier study (23 subjects, three lists each). The curves for the earlier study are shown with dotted lines. Under FR instructions (recall in any order), the curves are substantially the same as in the earlier study. Under SR instructions (recall in order of presentation), recall in $2-4$ lists is substantially lower than in the earlier study in all serial positions from 1 to 5 , whereas in 4-2 lists only positions 1 to 3 are affected. Under $F R(C)$ instructions (recall in groups related in meaning), recall in $2-4$ lists is substantially lower than in the earlier study in all serial positions except 1 and 6 , but in 4-2 lists recall is substantially higher in serial positions 1 to 4 .

The incidence of nonscorable words (repetitions or intrusions) was somewhat higher than in the earlier study; .63 per list in $4-2$ lists (.43) and .86 in $2-4$ lists (.55).

Analyses of variance were carried out on the scores obtained in the present experiment (two types of lists by three instruction conditions). On S1 scores only instructions were significant $(F=17.2, \quad d f=2 / 174$, $\mathrm{p}<.001)$. The mean score obtained was significantly lower under FR(C) instructions than under SR or FR instructions. On S2 scores both factors and their interaction were significant. Scores on 4-2 lists were higher than on $2-4$ lists $(F=67, d f=1 / 174, p<.001)$. SR instructions produced somewhat lower scores than FR or $F R(C)(F=4.74, d f=2 / 174, p<.025)$, and there was a complex interaction $(F=15.4, \quad d f=2 / 174$, 
$p<.001)$ in which, though the scores on $4-2$ and $2-4$ lists were much the same under FR instructions, SR and $F R(C)$ instructions produced higher scores than FR on 4-2 lists and the substantially lower scores already noted on 2-4 lists. On S3 scores both factors and their interaction were again significant. Scores on 4-2 lists were substantially higher than on 24 lists $(F=140$, $\mathrm{df}=1 / 174, \mathrm{p}<.001), \quad \mathrm{FR}(\mathrm{C})$ instructions produced substantially higher scores than $S R$ or $F R(F=46.2$, $\mathrm{df}=2 / 174, \mathrm{p}<.001)$, and there was an interaction $(F=8.02, \mathrm{df}=2 / 174, \mathrm{p}<.001)$ in which the increase from FR to $F R(C)$ instructions was much sharper in 4-2 lists than in $2-4$ lists.

SR instructions were less effective than FR(C) instructions in enhancing the corresponding type of score, which is consistent with the hypothesis.

\section{DISCUSSION}

The subjects in this experiment listened to each list knowing that they might be required to recall the words in the order of presentation or in groups related in meaning; they, therefore, had to attend to both aspects of the situation. When they were permitted to recall in any order (FR instructions), this extra load at the input stage did not seriously affect their S2 scores, which were as high as in the earlier study on 2-4 lists and not much lower on 4-2 lists. However, when they had to recall in order of presentation (SR instructions) or in groups of words related in meaning [FR(C) instructions], the $\mathrm{S} 2$ scores on $2-4$ lists were sharply reduced. This suggests that on $2-4$ lists the natural mode of recall relies on some characteristic of the word which is not related either to its semantic category or to its position in order. This is consistent with results of the earlier study which show that the S2 (total) score in 8-1 and 1-8 lists was always higher than the S1 (order) score, and the S2 (total) score in $2-4$ lists was always higher than the combined $S 1$ (order) and S3 (semantic) score. The 4-2 lists were, and are, exceptional in that the combined S1 and S3 score usually equals the S2 score.

Presenting SR instructions after 4-2 lists resulted in fewer words being recalled from the first three serial positions and more from the last five. Under FR(C) instructions, scores on the 4-2 lists were higher than in the previous study, but the advantage accrued entirely to words in the first four serial positions. It appears that, although the conditions of this experiment required the subject to pay less attention to the early items in the list than he would normally have been inclined to do (since he had to attend to both semantic and sequential characteristics), where only two semantic categories were involved, this entailed either no loss of efficiency or an actual gain [under FR(C) instructions]. Where four semantic categories were involved, there was, however, a loss of efficiency under SR and $\mathrm{FR}(\mathrm{C})$ instructions, though not (as we have seen) under FR instructions.

Perhaps memory may be conceptualized as a smooth surface on which verbal input causes transient "bumps" to appear and an attention process which recalls the word associated with any bump that has not subsided. More bumps will be processed in a given time if they are close together (i.e., if the words are semantically related) or if they have been sequentially tagged, since the attention process will waste less time on unproductive search. But some bumps may be located without the benefit of semantic or sequential clues.

In both types of lists and all three instruction conditions, S1 scores were substantially lower and S3 scores substantially higher than in the earlier study. This lends support to the suggestion that the general tendency to employ sequential characteristics observed in that study (which, when circumstances require, can clearly be overridden) may be related to the importance of word order as an indicator of meaning in English and many other languages. Overall, the subject appears to have a maximum memory capacity which he can deploy using sequential, semantic, or other criteria. The employment of words from just two semantic categories permits the subject to exhaust this capacity using semantic and sequential criteria only. Perhaps this fact stands in a relation of cause or effect to another characteristic of language-that propositions more frequently than not relate items from just two semantic categories!

\section{REFERENCES}

CraIK, F. I. M. A "levels of analysis" view of memory. In P. Pliner, L. Krames \& T. M. Allaway (Eds.), Communication and affect, language and thought (Vol. II). New York: Academic Press, 1973.

CRAIK, F. I. M., \& LockhaRT, R. S. Levels of processing: A framework for memory research. Journal of Verbal Learning and Verbal behaviour, 1972, 11, 671-684.

SAnders, A. F., \& Schroots, J. J. F. Cognitive categories and memory span: I. Shifting between categories. Quarterly Journal of Experimental Psychology, 1968, 20, 370-372.

WeTHERICK, N. E. The role of semantic information in shortterm memory. Journal of Verbal Learning and Verbal Behaviour, 1975, 14, 471-480.

(Received for publication April 7, 1976.) 